$$
\begin{array}{ll}
\text { trajectories, } & \text { if } F=\sqrt{W_{2}+h}, \\
\text { brachistochrones, } & \text { if } F=1 / \sqrt{W_{2}+h}, \\
\text { catenaries, } & \text { if } F=W_{2}+h .
\end{array}
$$

By comparison of (17) and (18), we may now state:

$A$ velocity system for the speed $\dot{s}_{0}$ in a conservative field with work function $W_{1}$ is a system of (1) trajectories, (2) brachistochrones, (3) catenaries for the constant of energy $h$ in a conservative field with work function $W_{2}$, where
(1) $W_{2}=e^{2 W_{1} / s_{0}^{2}}-h$,
(2) $W_{2}=e^{-\left(2 W_{1} / \dot{s}_{0}^{2}\right)}-h$,

(3) $W_{2}=e^{W_{1} / \dot{s}_{0}^{2}}-h$.

Since $W_{1}=$ constant gives $W_{2}=$ constant, the two fields have the same equipotential hypersurfaces and the same lines of force.

Massachusetts Institute of Technology, February, 1920.

\title{
AUGUSTUS DE MORGAN ON DIVERGENT SERIES.
}

BY PROFESSOR FLORIAN CAJORI.

(Read before the San Francisco Section of the American Mathematical Society April 10, 1920.)

Several English mathematicians writing in the second quarter of the nineteenth century disapproved of the banishment of divergent series which had been brought about by the followers of A. L. Cauchy and N. H. Abel. These protests were unheeded, doubtless because they were not accompanied by indications disclosing how divergent series could be used with safety. There was one exception, however: Augustus De Morgan reached results which, had they been followed up promptly, might have re-introduced divergent series thirty years earlier than was actually the case. De Morgan's researches have been overlooked in historical statements, except by $\mathrm{H}$. Burkhardt,* who, however, missed the parts of De Morgan which foreshadow a new theory.

* H. Burkhardt "Ueber den Gebrauch divergenter Reihen in der Zeit von 1750-1860," Math. Annalen, vol. 70 (1911), pp. 169-206. This article contains much minute information regarding many writers. 
Not only did De Morgan issue a vigorous protest against the abandonment of divergent series, but he laid the foundation to three parts of the subject as it stands to-day: (1) A principle of divergent series recently enunciated more clearly by G. H. Hardy; (2) a summation formula more general than that of Cauchy; (3) the use of asymptotic series.

Omitting many clever observations made by De Morgan, we confine ourselves to these three parts. There is considerable incompleteness in De Morgan's development of them, especially of the first part.

1. A Principle of Divergent Series.-De Morgan's point of view is partly contained in the following assumption*: "If then $V$ be expanded into the series $P_{0}+P_{1}+P_{2}+\cdots$ and if the sum of $n$ terms, $P_{0}+P_{1}+\cdots+P_{n-1}$ be called $Q_{n}$; we obviously have

$$
\int_{0}^{a} V d v=\int_{0}^{a} P_{0} d v+\int_{0}^{a} P_{1} d v+\cdots+\int_{0}^{a}\left(V-Q_{n}\right) d v,
$$

where $n$ is made infinite after integration. When the series $P_{0}+P_{1}+\cdots$ is convergent, then, even granting that $\boldsymbol{S}\left(V-Q_{\infty}\right) d v$ may have circumstances peculiar to $n=\infty$, it is of no consequence, since considerations of form are rendered useless by evanescence of value: the elements of $\int\left(V-Q_{n}\right) d v$ must, by the hypothesis of convergency, diminish without limit as compared with the corresponding elements of $\int P_{0} d v, \int P_{1} d v$, etc. Even if integration converted the convergent series into a divergent one, this would still be the case."

In this rather difficult passage, De Morgan considers

$$
\int_{0}^{a} \lim _{n \rightarrow \infty}\left(V-Q_{n}\right) d v \text { and } \lim _{n \rightarrow \infty} \int_{0}^{a}\left(V-Q_{n}\right) d v .
$$

If $P_{0}+P_{1}+\cdots$ is convergent, then, even if this infinite series becomes divergent on integration, he finds $\int_{0}^{a} \lim _{n \rightarrow \infty}\left(V-Q_{n}\right) d v=0$,

* A. De Morgan "On divergent series, and various points in analysis connected with them" in Cambridge Philosophical Society, Transactions, vol. 8, Part II, pp. 182-203; see page 189. The paper bears the date of Jan. 15, 1843; it was read March 4, 1844. De Morgan treated divergent series also in vol. 11 , pp. 190-202, of the above Transactions and in his Differential and Integral Calculus, London, 1842, chapters 19 and 20, but he did not reach noteworthy results other than those given in his article of 1844. 
and the elements of $\int_{0}^{a}\left(V-Q_{n}\right) d v$ must "diminish without limit" as " $n$ is made infinite after integration." It would seem that $\int_{0}^{a} \lim _{n \rightarrow \infty}\left(V-Q_{n}\right) d v$ is used here for the purpose of interpreting $\lim _{n \rightarrow \infty} \int_{0}^{a}\left(V-Q_{n}\right) d v$. No example is given of the conversion of a convergent into a divergent series by integration, but he takes

(1) $\frac{1-c \cos a v}{1-2 c \cos a v+c^{2}}=1+c \cos a v+\cdots+c^{n} \cos n a v+R_{n+1}$,

then "neglects" $R_{n+1}$ and lets $n=\infty$. Thus he obtains an infinite series which is divergent for $c>1$. Multiplying both sides by $e^{-v^{2}} d v$ and integrating from $v=0$ to $v=\infty$, he gets a convergent series. He shows this result to be arithmetically wrong.

Reversing the order of procedure and retaining $R_{n+1}$ in (1), he integrates first and then lets $n=\infty$; he obtains a convergent result which is arithmetically correct. We have here a distant approach to the principle advanced by G. H. Hardy* nearly sixty years later, to the effect that, in such cases, "we may use the otherwise meaningless expression" first obtained "as a formal equivalent for the determinate expression" obtained last.

2. A Summation Formula More General than that of Cauchy.That De Morgan in 1843 held views which were in advance of his time is evident from the following quotation: $\nmid$ "In every convergent series, the limit of the sum of all its terms is the mean value obtained from all the summations: the mean of $n$ partial summations $A_{1},\left(A_{1}+A_{2}\right), \cdots,\left(A_{1}+A_{2}+\cdots+A_{n}\right)$ is

$$
A_{1}+\frac{n-1}{n} A_{2}+\frac{n-2}{n} A_{3}+\cdots+\frac{1}{n} A_{n}
$$

which, as $n$ is increased without limit, has $A_{1}+A_{2}+\cdots$ ad inf. for its limit. Hence, by Poisson's principle, by which

* Cambridge Philosophical Society, Transactions, vol. 19, 1900-1904, p. 297. See also T. J. I. Bromwich, Infinite Series, London, 1908, p. 267, $\$ 99$.

$\dagger$ Cambridge Philosophical Society, Transactions, vol. 8, Part II, 1844, p. 192. 
I mean the assumption of the right to apply the maxim, 'that which is quantitatively true up to the limit, is true in the same sense at the limit, when the limit presents an incalculable form'-we may assert most positively, that $1-1+1-\cdots$ must be $\frac{1}{2}$ whenever it is the limiting form of convergency: not on the metaphysical doctrine (probably suggested by the known result) of Leibnitz, namely, that we can see no reason to prefer 0 to 1 , or 1 to 0 , and must therefore take a mean; but because $n$ partial summations give the mean $1 / n \times n / 2$ or $1 / n \times(n+1) / 2$ according as $n$ is even or odd, and the limit of both is $\frac{1}{2}$."

De Morgan's definition of "sum" is substantially the same as a definition given by G. Frobenius* in 1880 and the first of several definitions given by E. Cesàroł in 1890. De Morgan pointed out that, when applied to convergent series, his formula yields the same results as does the restricted formula ordinarily used for convergent series; that is, he recognized the need of "consistency" of definition.

3. The Use of Asymptotic Series.-As De Morgan's paper is dated January 15, 1843, he had not seen Cauchy's articleł printed later in the same year in which Stirling's divergent series for $\log \Gamma(x)$ is used for computing $\log \Gamma(x)$ for large positive values of $x$. De Morgan appreciates the importance of asymptotic series, but does not compute the asymptotic value of any one series as was done by Cauchy. Instead, De Morgan is groping after a general theory. He says:§ "When an alternating series is convergent, and a certain number of its terms are taken as an approximation, the first term neglected is a superior limit of the error of approximation. This very useful property was observed to belong to large classes of alternating series, when finitely or even infinitely divergent: I do not remember that any one has denied that it is universally true, while many have implicitly asserted it. When the series is convergent for a certain number of terms, particularly if the terms become very small before they begin to increase again, it obviously makes the divergent alternating

* G. Frobenius, "Ueber die Leibnitzsche Reihe," Jour. für Math., vol. 89 (1880), p. 262.

† E. Cesàro, "Sur la multiplication des séries," Bull. des Sciences Math. (2), vol. $14(1890)$, p. 119.

$\ddagger$ Cauchy, Comptes Rendus, vol. 17 (28 aout, 1843), p. 370; Euvres, Série I, vol. 8, p. 18.

§ Cambridge Philosophical Society, Transactions, vol. 8, Part II, p. 193. 
series practically as useful as the converging series, perhaps even more so, for it is very frequent that the greater the ultimate divergence, the greater also is the primitive tendency towards convergence."

The theorem that "the first term neglected is a superior limit of the error of approximation," though, as De Morgan says, not universally true, is true, he says, of large classes of alternating series, including the series $\phi(x)-\phi(x+1)$ $+\phi(x+2)-\ldots$ "for all cases in which $\phi(x)$ can be the expressed by $\int_{a}^{\beta} e^{n v x} X_{v} d v, X_{v}$ being always positive between limits."

In the development of the modern theories of divergent series, Augustus De Morgan deserves to be ranked as a pioneer.

On December 23, 1857, Sir William R. Hamilton* wrote to De Morgan: “About diverging series, you know a great deal more than I do. In fact you are aware that I early conceived a sort of prejudice against them, in consequence of some of Poisson's remarks. Counter-remarks of yours had staggered me, but had not been carefully weighed. At last (and, I regret to say it, without having yet found the Papers by you and Stokes on such series, for Stokes, or Adams for him, sent me about a month ago a duplicate of his memoir on the numerical calculation of the values of certain definite integrals, having a great affinity to my last Paper) I am become a convert to those Divergents; so far at least as to be satisfied that in an extensive class of cases, and with suitable limitations, they may be safely and advantageously used."

UNIVERSITY OF CALIFORNIA.

\section{RUSSELL'S INTRODUCTION TO MATHEMATICAL PHILOSOPHY.}

Introduction to Mathematical Philosophy. By Bertrand Russell. (The Library of Philosophy.) London, Allen and Unwin, and New York, The Macmillan Company, 1919. 8vo. viii +208 pp. $\$ 3.00$.

THIs book, called an introduction to mathematical philosophy, is an excellent introduction to that field and, more

* R. P. Graves, Life of Sir William Rowan Hanilton, vol. 3, 1899, p. 538. 